\title{
POSITIVE SOLUTION OF CRITICAL HARDY-SOBOLEV ELLIPTIC SYSTEMS WITH THE BOUNDARY SINGULARITY
}

\section{JIANFU YANG AND YIMIN ZHOU}

Abstract. In this paper, we are concerned with the existence of positive solutions to the system

$$
\begin{cases}-\Delta u=\frac{2 p}{p+q} u^{p-1} v^{q}+\frac{2 \lambda \alpha}{\alpha+\beta} \frac{u^{\alpha-1} v^{\beta}}{\mid x^{s}}, & \text { in } \Omega, \\ -\Delta v=\frac{2 q}{p+q} u^{p} v^{q-1}+\frac{2 \lambda \beta}{\alpha+\beta} \frac{u^{\alpha} v^{\beta-1}}{|x|^{s}}, & \text { in } \Omega, \\ u>0, v>0, & \text { in } \Omega, \\ u=v=0, & \text { on } \partial \Omega,\end{cases}
$$

where $\Omega$ is a $C^{2}$ domain in $\mathbb{R}^{N}$ with $0 \in \partial \Omega, 0<s<2, \lambda>0, p+q=2^{*}=\frac{2 N}{N-2}, \alpha+\beta=$ $2^{*}(s)=\frac{2(N-s)}{N-2}, N \geqslant 3$. We show that if $\Omega=\mathbb{R}_{+}^{N}$, problem (0.1) possesses a least energy solution and if $\Omega$ is bounded, $0 \in \partial \Omega$, there exists $\lambda^{*}>0$ such that problem (0.1) has at least a positive solution provided $0<\lambda<\lambda^{*}$.

Mathematics subject classification (2010): 35J47, 35J50, 35 J57.

Keywords and phrases: existence, positive solution, critical Hardy-Sobolev exponent, nonlinear system.

\section{REFERENCES}

[1] C. O. Alves, D. C. De Morais Filho And M. A. S. Souto, On systems of elliptic equations involving subcritical and critical Sobolev exponents, Nonlinear Anal. TMA, 42 (2000), 771-787.

[2] H. BréZis AND L. Nirenberg, Positive solutions of nonlinear elliptic equations involving critical Sobolev exponents, Comm. Pure Appl. Math., 36 (1983), 437-477.

[3] L. Ding And S.-W. XIAo, Solutions for singular elliptic systems involving Hardy-Sobolev critical nonlinearity, Diff. Equ. Appl., 2 (2010), 227-240.

[4] H. Egnell, Positive solutions of semilinear equations in cones, Trans. Amer. Math. Soc., 11 (1992), 191-201.

[5] N. GHoussoub AND X. S. KANG, Hardy-Sobolev critical elliptic equations with boundary singularities, Ann. Inst. H. Poincaré Anal. Non Linéaire, 21 (2004), 769-793.

[6] N. Ghoussoub And F. Robert, The effect of curvature on the best constant in the Hardy-Sobolev inequalities, Geom. Funct. Anal., 16 (2006), 1201-1245.

[7] N. Ghoussoub And F. ROBERT, Concentration estimates for Emden-Fowler equations with boundary singularities and critical growth, IMRP Int. Math. Res. Pap., 21867 (2006), 1-85.

[8] N. GHOUSSOUB AND F. ROBERT, Elliptic equations with critical growth and a large set of boundary singularities, Trans. Amer. Math. Soc., 361 (2009), 4843-4870.

[9] C. H. HSIA, C. S. LiN AND H. WADADE, Revisiting an idea of Brézis and Nirenberg, J. Funct. Anal., 259 (2010), 1816-1849.

[10] C.S. Lin AND H. WADADE, Minimizing problems for the Hardy-Sobolev type inequality with the sigularity on the boundary, preprint 2011.

[11] Y.-Y. Shang, C.-L. TANG, Positive solutions for Neumann elliptic problems involving critical Hardy-Sobolev exponent with boundary singularities, Nonlinear Anal. TMA, 70 (2009), 1302-1320.

[12] G. Talenti, Best constant in Sobolev inequality, Ann. Mat. Pura Appl., (4), 110 (1976), 353-372. 